\title{
MODELLING CROWDING EFFECTS IN INFECTIOUS DISEASE TRANSMISSION
}

\author{
EDWARD K. WATERS
}

(Received 4 May 2015; first published online 4 August 2015)

2010 Mathematics subject classification: primary 92D30; secondary 92D40, 92B05.

Keywords and phrases: infectious disease modelling, ecology, epidemiology.

Crowding is synonymous with patchy distributions, where some population units, called patches, contain more individuals than others. Lloyd's mean crowding index is a measure of crowding that has been used in differential equation models in ecology [1, 2]. In this thesis, a new mathematical justification of these models is provided (now published in [5]). The models are then adapted for use in infectious disease modelling. Two forms of Lloyd's mean crowding are proposed for use in an infectious disease modelling context: the number of susceptible individuals per infected individual per patch, $I_{I S}^{*}$, and the number of infected individuals per infected individual per patch, $I^{*}$.

It is shown that the value of $I_{I S}^{*}$ at the start of an epidemic gives the maximum number of transmission events per patch. Over the course of the epidemic, the value of $I^{*}$ increases towards this limiting value. The ratio of $I_{I S}^{*}$ and $I^{*}, \rho_{I}$, is therefore proposed as a measure of how efficiently infections are transmitted. A paper based upon these results is currently being prepared.

As available transmission events reduce with increasing values of $I^{*}$, disease becomes easier to eliminate and the coexistence of competing infections is facilitated. In response to these results, a vaccination threshold that accounts for patchy distributions of infected individuals is developed, resulting in lower proportions of the population needing to be vaccinated when $I^{*}$ increases in value. Human papillomavirus, a multi-strain sexually transmitted infection with a patchy distribution, is used to explore the implications of these findings in the real world. It is shown that vaccination targeting one strain can result in increases in infection with another, but also that a limited degree of cross protection against the nontarget strain can eliminate

Thesis submitted to the University of New South Wales in August 2014; degree approved on 27 November 2014; supervisors Harvinder Sidhu, Leesa Sidhu and the late Geoffry Mercer.

(c) 2015 Australian Mathematical Publishing Association Inc. 0004-9727/2015 \$16.00 
it, in keeping with the fact that patchy distributions make infections easier to eliminate. These results are published in [3].

Finally, the relationship between patch migration and crowding is shown. Changes in migration can result in either crowds of infected individuals and limited spread of infection, or the uniform spread of infection throughout the population. This final result demonstrates that understanding the movement of individuals is critical to the control of epidemics (see [4]).

\section{Acknowledgements}

The author acknowledges the support of his supervisors, Associate Professor Harvinder Sidhu, Dr Leesa Sidhu and the late Professor Geoffry Mercer, without whom it would not have been possible to complete this project.

\section{References}

[1] E. Kuno, 'Aggregation pattern of individuals and the outcomes of competition within and between species: differential equation models', Res. Popul. Ecol. 30(1) (1988), 69-82.

[2] M. Lloyd, 'Mean crowding', J. Anim. Ecol. (1967), 1-30.

[3] E. K. Waters, 'Aggregation and competitive exclusion: explaining the coexistence of human papillomavirus types and the effectiveness of limited vaccine conferred cross-immunity', Acta Biotheor. 60(4) (2012), 333-356.

[4] E. K. Waters, H. S. Sidhu and G. N. Mercer, 'Spatial heterogeneity in simple deterministic SIR models assessed ecologically', ANZIAM J. 54(1-2) (2012), 23-36.

[5] E. K. Waters, H. S. Sidhu, L. A. Sidhu and G. N. Mercer, 'Extended Lotka-Volterra equations incorporating population heterogeneity: derivation and analysis of the predator-prey case', Ecol. Model. 297 (2015), 187-195.

EDWARD K. WATERS, The University of Notre Dame Australia, School of Medicine, PO 160 Oxford St, Darlinghurst, NSW 2010, Australia e-mail: edward.waters.nsw@gmail.com 